\title{
Venous thromboembolism in coronary artery surgery
}

The incidence of deep vein thrombosis (DVT) after surgical operations ranges from $20 \%$ to $30 \%$ with a $2 \%$ incidence of non-fatal pulmonary embolus and a $0.8 \%$ incidence of fatal pulmonary embolism after general, orthopaedic, and urological surgery. ${ }^{1-5}$ The rate of thromboembolic events depends on factors in the patient, the type of operation performed, the urgency of the operation, and the use of perioperative subcutaneous heparin.

Despite the use of prophylactic measures, venous thromboembolism remains a problem. A recent report of the National Confidential enquiry into Perioperative Deaths recommends adherence to prophylactic protocols and further research. ${ }^{6}$

It has always been assumed that DVT after coronary artery surgery is rare because most patients with coronary artery disease (CAD) and unstable angina are usually heparinised right up to the time of surgery, and all patients undergoing open cardiac surgery are fully heparinised during the procedure for cardiopulmonary bypass.

There is little published information on the incidence of venous thromboembolic events after coronary artery bypass surgery or indeed any cardiac surgical operation. Factors that may predispose patients undergoing coronary artery grafting to the formation of DVT are:

- Restriction of activity because of unstable angina-13\% of bedridden patients have evidence of DVT on screening. ${ }^{\text {? }}$

- Recent myocardial infarcts-DVT was found in $10 \%-38 \%$ of patients who had had a myocardial infarction. ${ }^{7}$

- Smoking is a risk factor for both coronary artery disease and DVT.

- In surgical thromboembolic disease, age is the most important risk factor, ${ }^{8}$ with the risk rising steeply after the age of 40 years. In recent years the mean age of patients undergoing cardiac surgery has risen steadily so that many patients undergoing coronary surgery are over 65 .

- In the postoperative period, extensive wounds in the legs and resultant restriction of limb activity may result in thrombus formation in the deep veins.

The diagnosis of venous thromboembolism is not entertained after coronary surgery because of preconceived ideas about the risk and because swelling of the legs and hypoxia are frequently present in the early postoperative period.

Anecdotal evidence and a recent report ${ }^{9}$ suggest that venous thromboembolism may not be rare after coronary artery surgery. During one 12 month period at Papworth Hospital, there were seven cases of fatal or clinically significant pulmonary emboli in 1400 patients undergoing open heart surgery $(0.5 \%)$. Six $(0.7 \%)$ of these patients had undergone coronary artery bypass grafting out of a total of 875. Two hundred and twenty five out of the total of 1400 patients operated on had been bedbound for several days. Five of the seven fatal and clinically significant pulmonary emboli occurred in this group - the pulmonary embolism rate was therefore $5 / 225(2 \cdot 2 \%)$ compared with two out of 1175 non-urgent cases $(0.17 \%)(P<0.002$, Fisher's exact test) - a rate comparable to other forms of surgery. ${ }^{1}$ As in other forms of surgery, prolonged bed rest is therefore a risk factor for the development of fatal or clinically significant pulmonary embolus after coronary artery surgery despite the fact that four of the patients were treated with intravenous heparin before operation.

In a recent paper Reis et al used high resolution $B$ mode ultrasonography to examine the frequency of DVT in symptom free patients after coronary artery surgery. ${ }^{9}$ They found DVT in $48.3 \%$. Only 29 patients were examined, and the $95 \%$ confidence interval was wide (30 to $66 \cdot 4 \%$ ). In addition there was no indication of the number of preoperative DVTs present nor their clinical consequence. The incidence of DVTs and non-fatal pulmonary embolus after coronary artery surgery is not known and a properly conducted prospective study is required.

At Papworth Hospital, we plan to start a prospective study by screening for DVT all patients going for coronary artery surgery. We will be using high resolution Doppler ultrasound with colour flow as the screening method. In a recent paper, Nicolaides and Kalodiki suggested that duplex scanning with colour flow will become the noninvasive method to screen symptom free patients for DVT. ${ }^{10}$

In conclusion, the incidence of venous thromboembolism after coronary artery surgery is unknown. Because the cohort of patients known to be at risk of this complication - that is, the elderly with unstable angina-is increasing, it is important that further studies are carried out. If the incidence of DVT is higher than previously thought, more attention must be directed towards prophylactic measures in this high risk group.

NORMAN BRIFFA

Surgical Unit STEPHEN R LARGE

Papworth Hospital NHS Trust, Cambridge CB3 8RE

1 Prevention of fatal postoperative pulmonary embolism by low doses of heparin: an international multicentre trial. Lancet 1975;ii:45-51

2 Kiil J, Axelson F, Andersen D. Prophylaxis against postoperative pulmonary embolism and deep vein thrombosis by low dose heparin. Lancet 1978; $1115-6$.

3 Williams JW, Eikman EA, Greenberg SH, Hewitt JC, Lopez Cuence E, Jones GP, Madden JA. Failure of low dose heparin to prevent pulmonary embolism after hip surgery or above knee amputation. Ann Surg 1978; 188:468-74.

4 Morris GK, Henry APJ, Preston J. Prevention of deep vein thrombosis by low dose heparin in patients undergoing total hip replacement. Lancet 1974;ii:795-7.

5 Coe NP, Collins REC, Klein LA, Bettmann MA, Skillman J, Shapiro RM, Salzman EW. Prevention of deep vein thrombosis in urologic patients: a controlled, randomised trial of low dose heparin and external pneumatic compression boots. Surgery 1978;83:230-4.

6 Campling EA, Devlin HB, Hoile RW, Lunn JN. The report of the national confidential enquiry into perioperative deaths. 1991/2. National Con-

7 Grace R, Hunt B. Thromboprophylaxis. Br f Hosp Med 1993;49:720-6.

8 Nicolaides AN, Irving MW. Clinical factors and the risk of deep vein thrombosis. In: Nicolaides A, ed. Thromboembolism aetiology-Advances in
prevention and management

9 prevention and management. Lancaster: MTP, 1975:193-204. Goldhaber SZ. Frequency of deep vein thrombosis in asymptomatic Goldhaber SZ. Frequency of deep vein thrombosis in asympto
patients with coronary artery bypass. Am Heart $f$ 1991;122:478-82.

10 Nicolaides A, Kalodiki E. Duplex scanning in postoperative surgical patients. Haemostasis 1993;23(suppl 1):72-9. 